\title{
Constraints on lithospheric mantle and crustal anisotropy in the NoMelt area from an analysis of long-period seafloor magnetotelluric data
}

Tetsuo Matsuno ${ }^{1,2^{*}}$ (iD and Rob. L. Evans ${ }^{3}$

\begin{abstract}
Despite strong anisotropy seen in analysis of seismic data from the NoMelt experiment in $70 \mathrm{Ma}$ Pacific seafloor, a previous analysis of coincident magnetotelluric (MT) data showed no evidence for anisotropy in the electrical conductivity structure of either lithosphere or asthenosphere. We revisit the MT data and use 1D anisotropic models of the lithosphere to demonstrate the limits of acceptable anisotropy within the data. We construct 1D models by varying the thickness and the degree of anisotropy within the lithosphere and conduct a series of tests to investigate what types of electrical anisotropy are compatible with the data. We find that electrical anisotropy is possible in a sheared and/or hydrous mantle within the lower lithosphere (60-90 km depth). The data are not compatible with pervasive electrical anisotropy in the crust. Causes of anisotropy within the highly resistive upper and mid-lithosphere, as seen seismically, are not expected to cause measurable impacts on MT response.
\end{abstract}

Keywords: Electrical anisotropy, Oceanic lithosphere, Shearing, Water, Central Pacific

\section{Introduction}

The formation of lithosphere at a mid-ocean ridge and the subsequent movement of that lithosphere across the underlying convecting asthenosphere result in deformation through shearing. This deformation can result in textural changes (lattice preferred orientation, crystal preferred orientation, and shape preferred orientation) of rocks that may affect their physical properties, such as seismic velocity, electrical conductivity, and viscosity. It is well known that lattice preferred orientation of olivine can be imaged seismically (e.g., Tanimoto and Anderson 1984; Nicolas and Christensen 1987; Montagner and Guillot 2002). If well imaged, patterns and depth distribution of seismic anisotropy can be used to constrain patterns of flow either occurring at present in the asthenosphere or in the past, preserved as the fabric frozen

\footnotetext{
*Correspondence: matsuno@port.kobe-u.ac.jp

1 Kobe Ocean Bottom Exploration Center, Kobe University, Kobe, Japan Full list of author information is available at the end of the article
}

into the lithosphere, and accordingly to provide insight into models of lithospheric formation and evolution (e.g., Blackman and Kendall 2002).

There are conflicting models of seismic anisotropy in the Pacific basin. Some models, derived from global seismic data sets, have suggested a relatively isotropic lithospheric structure underlain by an asthenosphere with substantial anisotropy resulting from current mantle flow (e.g., Beghein et al. 2014). More localized seismic results from the NoMelt experiment, carried out over 70 Ma lithosphere in the central Pacific, show strong fast azimuthal anisotropy within the lithosphere in a direction aligned parallel to that of past spreading at the ridge crest (Lin et al. 2016). The NoMelt seismic data also show more modest azimuthal anisotropy in the asthenosphere with the weakest anisotropy seen in the middle of the seismic low-velocity zone ( 100-150 km depth) before increasing again ( $200 \mathrm{~km} \mathrm{depth}$ ) (Lin et al. 2016).

Alignment of olivine $a$-axes through mantle flow results in the primary signal of seismic anisotropy (e.g., 
Blackman et al. 2002; Blackman and Kendall 2002). Until recently, the understanding has been that dry olivine has a low degree of electrical anisotropy, at most a factor of 2 (e.g., Du Frane et al. 2005; Yoshino et al. 2006; Poe et al. 2010), which is essentially undetectable by magnetotelluric (MT) sounding. Under these conditions, we would not expect significant electrical anisotropy in the lithospheric mantle.

Recent work shows that if olivine is sheared or is hydrous, electrical anisotropy can be generated. Pommier et al. (2015; pers. comm.) have shown that if there is significant shearing in the upper mantle, as might be expected at the base of the lithosphere or through flow at a mid-ocean ridge, substantial differences in conductivity can be expected between the direction parallel to shear and the orthogonal direction via grain boundary conduction mechanisms even for melt-free and dry samples. The effect is up to 0.5 order of magnitude at shallow lithospheric temperatures $\left(<\sim 900{ }^{\circ} \mathrm{C}\right)$ at strains of $1.3-7.3$ (Pommier et al. 2015; pers. comm.). The effect of adding water to olivine is more controversial, but there is some evidence that hydrous olivine with $\sim 100-400 \mathrm{ppm}$ water content shows an anisotropy of $\geq \sim 0.5$ order of magnitude at the upper mantle temperature (Gardés et al. 2014; Dai and Kararo 2014).

Despite the strong patterns of anisotropy seen in the NoMelt seismic data and possible electrical anisotropy due to shearing and hydration in the lithosphere, a previous analysis of coincident MT data showed no evidence for electrical anisotropy in the lithosphere, as well as the asthenosphere (Sarafian et al. 2015). This observation raises two questions. (1) Could the MT data detect electrical anisotropy at any depth within the lithosphere at the NoMelt region? (2) Would anisotropy be compatible with the NoMelt observation and, if so, what constraints can we place on the properties of the mantle such as shearing, hydration, and thermal structure? To answer these questions, here we revisit those data and use layered 1D anisotropic electrical conductivity models to demonstrate the limits of acceptable electrical anisotropy within the data and further explore implications on anisotropy in the lithosphere in the NoMelt area.

Our investigation of lithospheric electrical anisotropy is conducted using the following workflow. We start with the 1D isotropic model of Sarafian et al. (2015), which was a lateral average of a 2D isotropic model compatible with the data collected. We impose anisotropy onto this base 1D model in three ways to construct hypothetical 1D anisotropic models. Then, we conduct 1D forward modeling with the anisotropic models and sum the splits in the phases of the off-diagonal tensor elements. Thus, the sum of phase splits is used as a diagnostic tool to investigate where anisotropy within the lithosphere has the greatest impact of MT responses. We finally invert the synthetic $1 \mathrm{D}$ anisotropic responses using a $2 \mathrm{D}$ isotropic inversion algorithm to examine whether and how these anisotropic structures would be manifest in isotropic analysis.

Before we proceed to our study, we first confirm the NoMelt data (Additional file 1: Figure S1) and modeling of Sarafian et al. (2015) (Additional file 1: Figures S2 and S3), in particular, the assertion by Sarafian et al. (2015) that anisotropy is not a required feature of the data. The data at four sites along $\sim 600 \mathrm{~km}$ transect show weak lateral variations, and only a modest amount of splitting between off-diagonal elements is seen in phase $\left(<5^{\circ}\right)$ and apparent resistivity $(<0.2$ in logarithmic scale). Typical standard errors are a few degrees for the off-diagonal phase and $<0.1$ in logarithmic scale for the off-diagonal apparent resistivity.

We reconfirmed that electrical anisotropy in the lithosphere and asthenosphere that is inferred from 2D anisotropic inversions is weak by running a series of inversion using the same 2D inversion program as Sarafian et al. (2015) with a wider range of regularization parameters for model smoothing $\left(\tau_{\mathrm{s}}\right)$ and anisotropy $\left(\tau_{\mathrm{c}}\right)$ than Sarafian et al. (2015) (both $\tau_{\mathrm{s}}$ and $\tau_{\mathrm{c}}$ ranged from $10^{-2}$ to $10^{2}$ with increments of 0.5 order of magnitude). A resulting $2 \mathrm{D}$ anisotropic model does not show strong anisotropy in either lithosphere or asthenosphere $(\sim 0.2$ in logarithmic scale) and do not provide significant improvement in data misfit in comparison with the 2D isotropic model (Additional file 1: Figures S2 and S3). Although statistically the fits to the data that are achieved by this process are acceptable, there is some residual misfit, especially in TE-mode phases at periods less than $10^{4} \mathrm{~s}$, for the isotropy and anisotropy models. This residual misfit could be the result of off-profile and/or small-scale heterogeneity around each station. We are satisfied that these residuals cannot be explained by anisotropy in a 2D framework and do not further investigate such heterogeneities in this study. We also confirmed that any rotations of the coordinate system for MT responses used in the inversion did not yield strong electrical anisotropy in the resultant inversion models. Thus, the starting point for our modeling is the 1D isotropic resistivity profile of Sarafian et al. (2015).

\section{Forward modeling and inversion tests}

We constructed a series of 1D anisotropic models that are based around the background 1D profile (Sarafian et al. 2015) and conducted forward modeling and inversions tests. Model parameters changed from the 1D profile are the thickness of an anisotropic layer $(h)$ between a surface isotropic layer and a depth of $90 \mathrm{~km}$ that is consistent with the bottom of lithosphere (Sarafian et al. 
$2015)$, and the degree of anisotropy $(\alpha)$ of the anisotropic layer (Fig. 1).

In constructing synthetic anisotropic 1D models, we consider a model of lithosphere formation where a compositional lithosphere is formed at the mid-ocean ridge with $\sim 60 \mathrm{~km}$ thickness, consisting of a depleted and dry residual mantle (Evans et al. 2005; Baba et al. 2006), with potentially complex fabric depending on flow patterns at ridge. Strong azimuthal anisotropy in the NoMelt upper mantle that decreases with depth through the lithosphere (Lin et al. 2016) suggests a greater degree of shearing and fabric alignment at shallow depths. We also suggest that the lowermost lithosphere $(\sim 60-90 \mathrm{~km})$ in the NoMelt $70 \mathrm{Ma}$ seafloor area, which is thicker than at the midocean ridge of $\sim 60 \mathrm{~km}$, could be damp and sheared and have accreted onto the base of the overlying plate with age. This layer would have a fabric anisotropy associated with plate motion at the time of accretion and so could also be electrically anisotropic by shearing. The water content of the layer would further enhance its conductivity and also potentially result in electrical anisotropy. These effects could work in isolation or together, but in any event we might expect a layer toward the base of the lithosphere with significant electrical anisotropy.
In generating and testing models, an important consideration is how the anisotropy is applied to the background 1D isotropic profile. There are three ways to impose anisotropy (Fig. 1). The first maintains the background model as the most conductive direction and forms a more resistive model (by a factor of $\alpha$ ) for the orthogonal direction (type 1). The second approach is to split the anisotropy in half between the two axis directions by subtracting resistivity from one direction and adding to the other such that the total conductivity split is a factor of $\alpha$, centered on the background profile (type 2 ). The third possibility is the opposite of type 1 , where the background model follows the most resistive direction and the imposed anisotropy results in a layer that is more conductive (type 3). Because MT is less sensitive to resistive layers, changes to the model to make it more conductive have a more significant and pronounced effect on the response. Figure 1 shows examples of these three approaches ( $h=85 \mathrm{~km}$ and $\alpha=1.0)$.

For each model type, we have generated a suite of responses by varying $h$ and $\alpha$ through forward modeling for 1D layered anisotropic electrical conductivity structures (Pek and Santos 2002). We assume that the most conductive direction $(y$-direction for MT

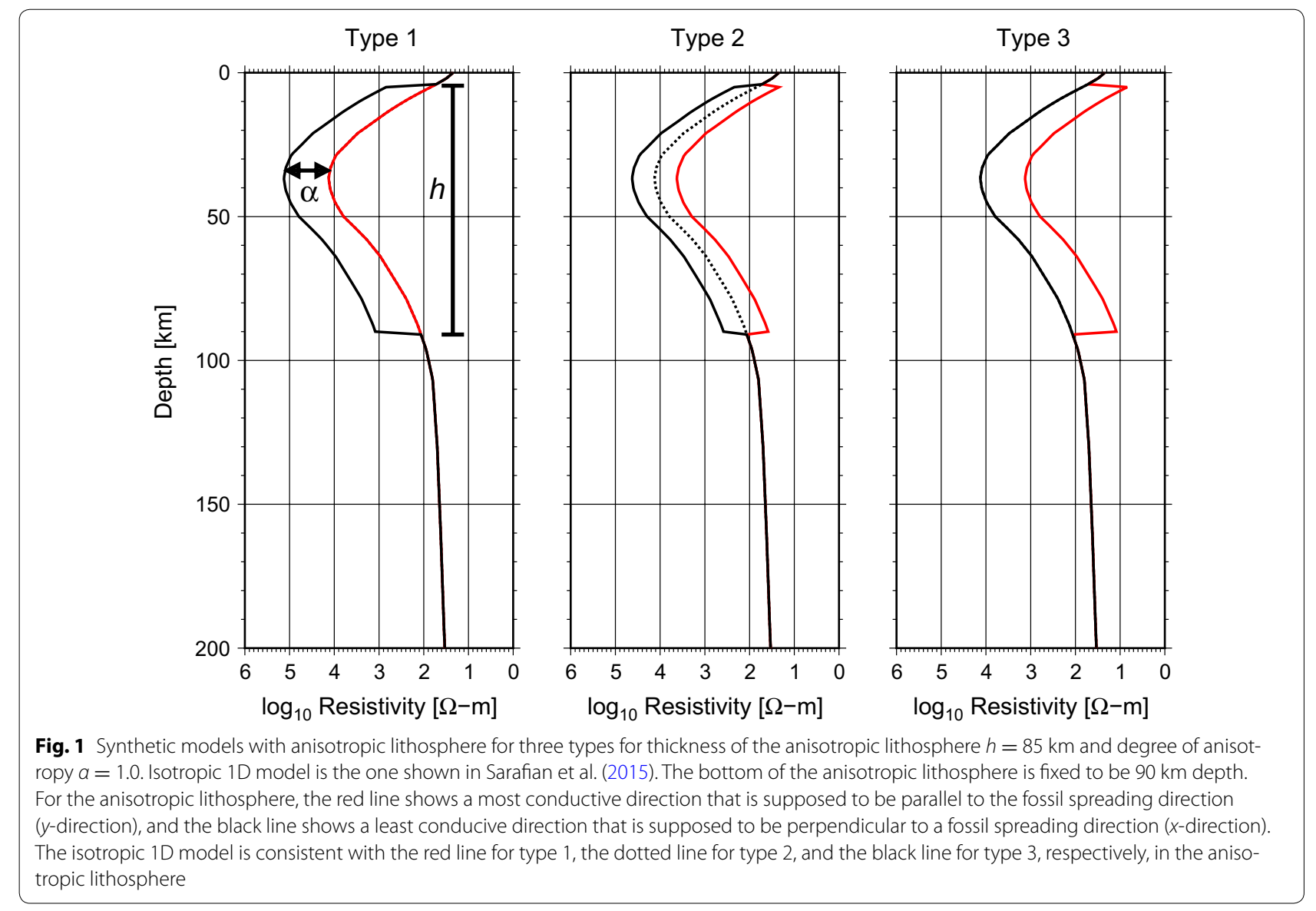


response) corresponds to the fossil spreading direction $\left(\sim \mathrm{N} 80^{\circ} \mathrm{E}-\mathrm{S} 80^{\circ} \mathrm{W}\right)$ and least conductive ( $x$-direction for MT response) is parallel to the strike of the paleo-ridge $\left(\sim \mathrm{N} 10^{\circ} \mathrm{W}-\mathrm{S} 10^{\circ} \mathrm{E}\right)$ (Lin et al. 2016). The seismic data show clear evidence for anisotropy in the fossil spreading direction (Lin et al. 2016), so we maintain this geometry in assigning anisotropy.

These 1D anisotropic models predict large splits in phase at periods from $\sim 100$ to $10^{4} \mathrm{~s}$ (Fig. 2). We then calculate the sum of the phase split for 8 periods from 640 to $7680 \mathrm{~s}$, consistent with the period band over which stable MT responses with small errors were obtained (Sarafian et al. (2015) and Additional file 1: Figure S1). For the NoMelt data responses, the cumulative phase splits over this period range are $35^{\circ}, 8^{\circ}, 21^{\circ}$, and $18^{\circ}$ for the four sites M01, M02, M04, and M06, respectively (Sarafian et al. 2015). We assume a cumulative split of $20^{\circ}$ is representative of the data. The suites of summed phase splits for synthetic models define a surface as a function of $h$ and $\alpha$ (Fig. 3). Maps of splitting in phase show a clear trend for the influence of $h$ and $\alpha$; larger $h$ and $\alpha$ result in larger splitting in phase. There is also a trend between model types; the phase splitting is larger as the anisotropic structure becomes more conductive than the background model, because of the higher sensitivity of the MT data to conductive layers.

There are splits in observed apparent resistivities of the NoMelt data (Sarafian et al. 2015) (Additional file 1:
Figure S1). Split in apparent resistivity provides a better constraint on anisotropic structures than does phase (Heise et al. 2006). However, the phase is more robust to distortion of static shift than apparent resistivity that could be found in the NoMelt data. The sense of splitting in apparent resistivity is opposite for the model prediction ( $x y$ element is larger than $y x$ element) (Fig. 2) than for the observation ( $y x$ element is larger than $x y$ element) (Additional file 1: Figure S1). If we switch the most conductive direction and the least conductive direction in the models (i.e., $x$-direction is the most conductive and $y$-direction is the least conductive), however, the sense of splitting is switched not only in apparent resistivity but also in phase, as expected. This phase split is the opposite sense to the real data (Additional file 1: Figure S1). Therefore, we consider only splits in phase as a more robust diagnostic in this study.

Synthetic data generated from the 1D anisotropic models have only off-diagonal elements because the coordinate system for MT response is aligned to the geometry of the anisotropic structures. Even then, off-diagonal elements are split due to structural anisotropy, so we applied a 2D isotropic inversion program to the $1 \mathrm{D}$ anisotropic data to see which of the resulting inversion models of each model type most closely resembles the 1D background profile onto which anisotropy is imposed. The 2D inversion code used is based on Rodi and Mackie (2001) and can deal with transversely anisotropy in the
Type 1
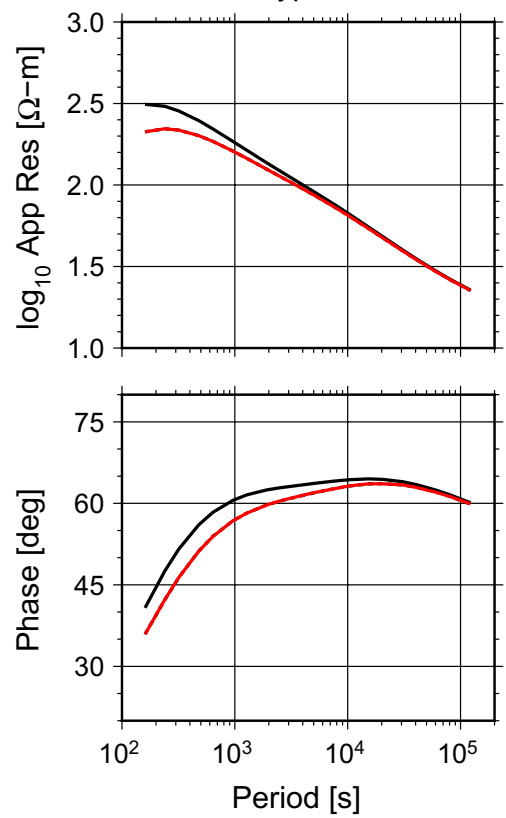

Type 2
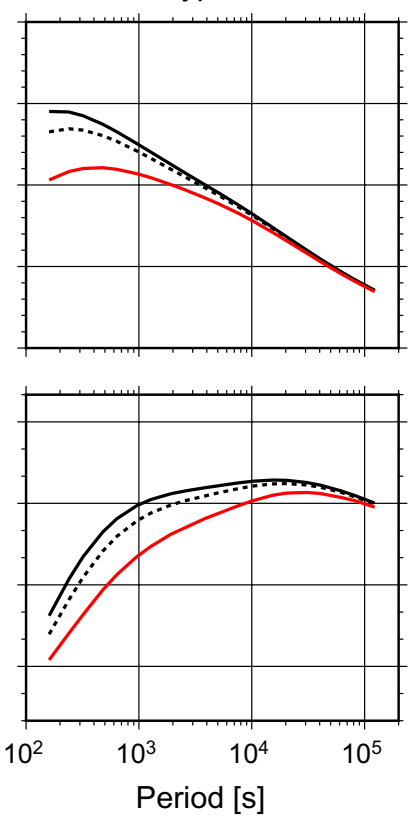

Type 3
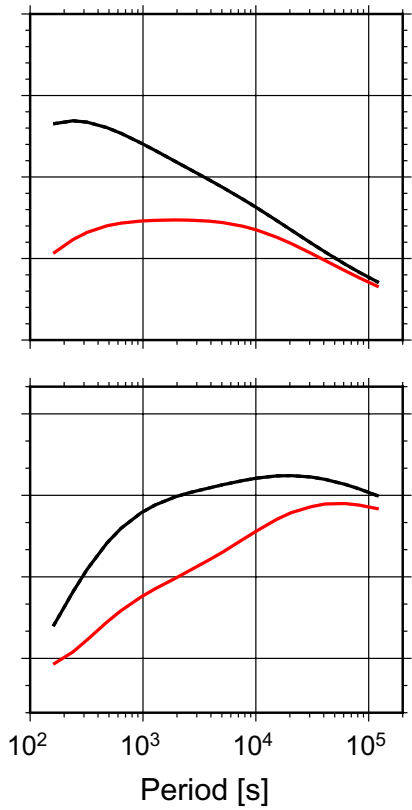

Fig. 2 Off-diagonal MT responses with splitting for three synthetic anisotropic models shown in Fig. 1. Red is most conductive element (yx), and black is least conductive element (xy). Dotted line in the type 2 plot shows responses of the isotropic 1D model of Sarafian et al. (2015) 


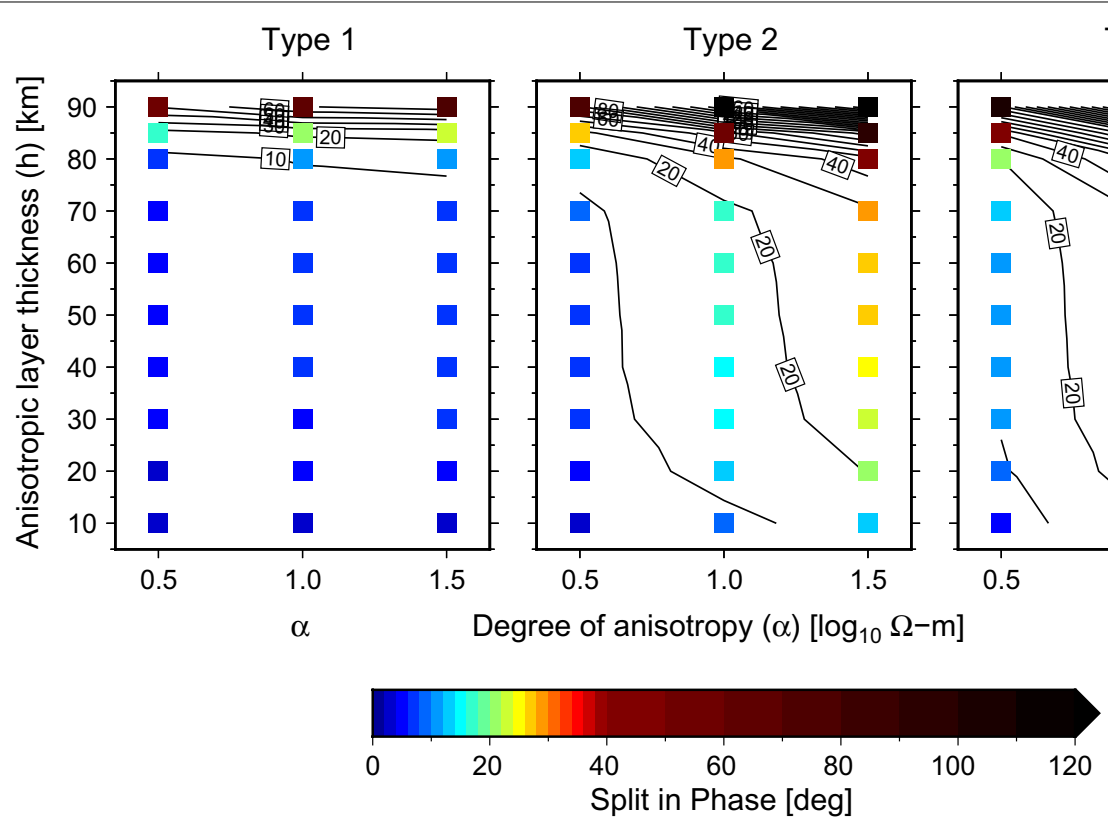

Type 3

Fig. 3 Maps of cumulative phase splitting between off-diagonal ( $y x$ and $x y$ ) elements at 8 periods from 640 to $7680 \mathrm{~s}$ as a function of the anisotropic layer thickness $(h)$ and the degree of anisotropy $(a)$

2D model (Baba et al. 2006). However, we do not use the full functionality of the code but use it for obtaining $2 \mathrm{D}$ isotropic models. We force any inversion model to be isotropic by setting the regularization parameter for anisotropy to a value of $10^{6}$. We did not give scatter to the synthetic inversion data, but applied an error floor of $1 \%$ to apparent resistivity and phase $\left(0.3^{\circ}\right)$ in the inversion. We pick only one 1D profile immediately below a site from the resulting 2D isotropic model.

We have run a series of isotropic inversions of the synthetic data for each of the three model types and show an example of the case for $h=85 \mathrm{~km}$ and $\alpha=1.0$ (Fig. 4). Regularization parameters for model smoothness were determined by L-curve tests to be 0.1 for types 1 and 3 and 0.3 for type 2 . Results show that inversion models for types 1 and 2 are consistent with the 1D isotropic model of Sarafian et al. (2015) at shallow depths $(<20 \mathrm{~km})$ and in the asthenosphere $(>90 \mathrm{~km})$, with differences seen in the mid-lithosphere between depths of $20-60 \mathrm{~km}$. The similarity of the inversion models between types 1 and 2 suggests that we cannot distinguish these models from an isotropic $2 \mathrm{D}$ inversion of $1 \mathrm{D}$ anisotropic MT data. The inconsistency in the lithospheric structure that is predominantly in 20-60 km depth is due to the lower resolving capability of MT to resistive structure as well as a model produced artificially by regularization with model smoothing. In contrast, an inversion of type 3 model data is biased toward recovering the most conductive profile in the top to the middle of the lithospheric structure, but does a reasonable job of finding the background structure in the lowermost lithosphere.

\section{Comparison with the observation and implications for lithospheric structure}

We stress that we assume that a representative cumulative phase split at periods from 640 to $7680 \mathrm{~s}$ in the NoMelt observational data is $20^{\circ}$. For simplicity, when evaluating phase splits we have not taken response errors into consideration (typically $1^{\circ}-4^{\circ}$ for the $x y$ element and $0.5^{\circ}-2^{\circ}$ for $y x$ element in standard error; Sarafian et al. (2015) and Additional file 1: Figure S1). If the errors are considered, the amount of cumulative splitting in data to be confident of inferring anisotropy would clearly need to be larger.

With the synthetic model of type 1, forward modeling splits suggest that an electrical anisotropy up to 1.5 order of magnitude would be allowable over virtually the whole lithospheric mantle (up to $h=85 \mathrm{~km}$ ) with the assumption for the representative cumulative split of $20^{\circ}$ (Fig. 3). Inversion of type 1 model data returns a profile that is broadly consistent with the isotropic 1D model (Sarafian et al. 2015), except in the mid-lithosphere (20-60 km depth) (Fig. 4). This result suggests that 2D inversion of data sets from type 1 anisotropic structures results in the isotropic 1D model of Sarafian et al. (2015). Thus, the observed splitting could be consistent with a type 1 model with strong anisotropy over the whole lithospheric mantle. The mechanisms responsible for generating 

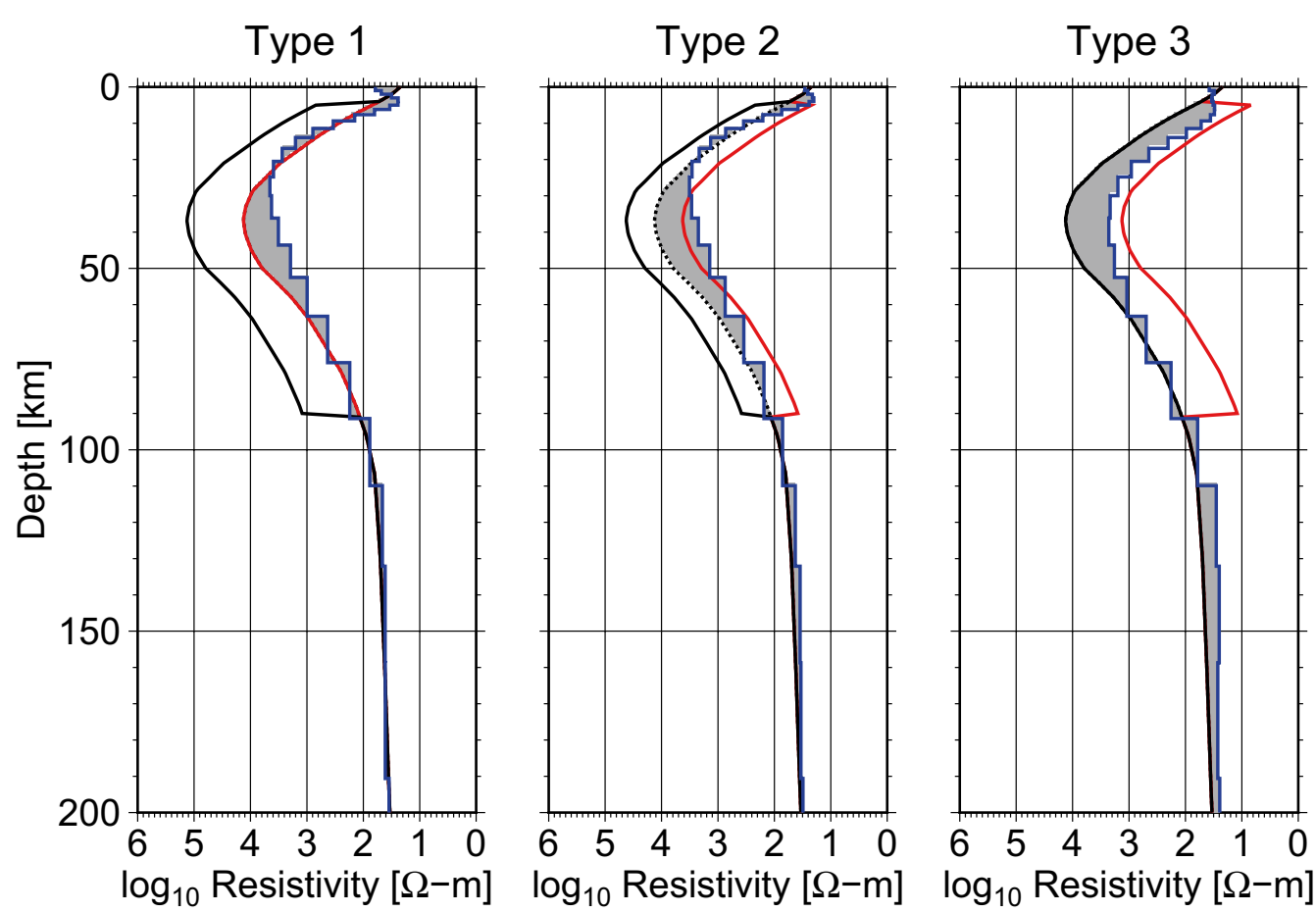

Fig. $42 \mathrm{D}$ isotropic inversion results (blue lines) of 1D anisotropic data produced from three types of synthetic true models. Discrepancy between the 2D isotropic inversion model and the true 1D isotropic model is shaded in each plot. The synthetic true models are same as Fig. 1

electrical anisotropy in the lithospheric mantle should act to enhance conductivity in the direction of shearing or fabric alignment of olivine, and the least conductive profile through the lithosphere should represent the geotherm in a dry lithospheric mantle. The isotropic 1D profile in the asthenosphere in the NoMelt area is basically an adiabatic profile $\left(0.3^{\circ}-0.5^{\circ} \mathrm{C} / \mathrm{km}\right)$ with, at most, water contents consistent with MORB source geochemistry (Sarafian et al. 2015). To realize type 1 anisotropic model profiles, temperature in the lithosphere should be lower than this asthenospheric geotherm. Assuming a dry olivine composition (e.g., Constable 2006), higher resistivity by half an order of magnitude requires a reduction in temperature $\sim 100{ }^{\circ} \mathrm{C}$. The way we have constructed our models suggests a thermal discontinuity at the lithosphere-asthenosphere for model type 1 , but this transition could be smoother than we have considered.

For model type 3, the very large cumulative phase splits seen in forward modeling suggest that electrical anisotropy is not compatible with observations if there is significant anisotropy in the crust and the uppermost mantle ( $h>80 \mathrm{~km}$ ) extending through the lithosphere, and also for $\alpha>0.5$ (Fig. 3). Models generated from inversions of data from this class of model are inconsistent with the isotropic 1D model, especially in mid-lithosphere, where the inversion model follows the most conductive profile. This suggests that $2 \mathrm{D}$ inversions of data from type
3 anisotropic models result in 1D models that deviate from the isotropic 1D model (Sarafian et al. 2015). However, the inversion test suggests that in the lower lithosphere, for this class of anisotropy, the 1D profile would well represent the background conductivity, with the most conductive direction not sensed. These tests show that only weak anisotropy in a type 3 model is compatible with the NoMelt data, but that some level of anisotropic conductivity enhancement is permissible in the lower lithosphere.

The type 2 model result lies between those for the type 1 and 3 models in forward modeling (Fig. 3). A contour of $20^{\circ}$ of cumulative phase splitting delineates the upper right area of the parameter plot (Fig. 3). Inversion tests are similar to those for the type 1 model and appear to trace the most conductive profile in mid-lithosphere but are consistent with the average profile in the lower lithosphere. Based on the same considerations on the thermal profile and anisotropy mechanisms as the other type of models, electrical anisotropy of 0.5 order of magnitude is consistent with not only in the lower lithosphere but also in the middle- and upper lithosphere.

While electrical anisotropy in the crust should have an impact on MT responses as we showed in this study, observations of crustal anisotropy, and on the uppermost-mantle anisotropy, are controversial. There is one good observation to suggest that the oceanic crust is 
electrically isotropic, except when the plate is bent at a subduction zone in Middle America (Key et al. 2012; Naif et al. 2015). The crust is more conductive parallel to the trench axis and the fossil seafloor spreading axis in the plate bending area by a factor of $5\left(6 \times 10^{2} \Omega \mathrm{m}\right.$ and $3 \times 10^{3} \Omega \mathrm{m}$ or $1.7 \times 10^{-3} \mathrm{~S} / \mathrm{m}$ and $3.3 \times 10^{-4} \mathrm{~S} / \mathrm{m}$ ) (Key et al. 2012; Naif et al. 2015). Another data set in $40 \mathrm{Ma}$ lithosphere (Everett and Constable 1999), which has less complete azimuthal coverage, suggests higher conductivity (by a factor of 7, $1.4 \times 10^{3} \Omega \mathrm{m}$ and $1 \times 10^{4} \Omega \mathrm{m}$ or $7 \times 10^{-4} \mathrm{~S} / \mathrm{m}$ and $1 \times 10^{-4} \mathrm{~S} / \mathrm{m}$ ) in the uppermost mantle aligned in the direction of fossil plate spreading. This result is consistent with that expected if shearing and/or hydrous $a$-axis aligned olivine dominate the conductivity mechanism in the uppermost mantle. On the other hand, there is the possibility that cracks and faults formed at the mid-ocean ridge induce an electrical anisotropy with the most conductive direction parallel to the ridge. This seems to be the observation of Behrens (2005), who suggests higher conductivity (by a factor of $2-3,1 \times 10^{4} \Omega \mathrm{m}$ and $2-3.3 \times 10^{4} \Omega \mathrm{m}$ or $1 \times 10^{-4} \mathrm{~S} / \mathrm{m}$ and $3-5 \times 10^{-5} \mathrm{~S} / \mathrm{m}$ ) parallel to the paleo-ridge direction, although that study was unable to constrain the depth distribution of anisotropy.

Our tests exclude the possibility of crustal anisotropy that is conductive in the strike direction of the paleoridge. In addition, we tested the impacts of including crustal anisotropy orthogonal to the underlying anisotropy in the lithospheric mantle. This new test revealed that splits between off-diagonal phases could be reduced by an effect of masking the underlying mantle anisotropy by the crustal anisotropy. However, the modeled splits are still larger than the NoMelt observation of $\sim 20^{\circ}$, so the NoMelt data do not support crustal anisotropy with most conductive aligned with the strike of the paleoridge. This implies that electrical anisotropy does not exist in the crust near the NoMelt sites. This is probably because electrical anisotropy in the crust is not pervasive but localized such as at faults if existed.

From these tests, and also taking into consideration observed seismic anisotropy in the lithosphere in the NoMelt area, we draw the following conclusions:

- It is possible that there is electrical anisotropy in the lowermost lithosphere $(60-90 \mathrm{~km})$ by $\geq 0.5$ order of magnitude. This could be compatible with the presence of modest amount of water in the lower lithosphere and/or a highly sheared lithospheric structure in melt-free olivine mantle, where seismic azimuthal anisotropy was found to be oriented to the fossil spreading direction.

- Electrical anisotropy could begin beneath the moho and extends through the lithospheric mantle and then might be consistent with the existence of the strong seismic azimuthal anisotropy observed in this area. The degree of anisotropy is dependent on the lithospheric temperature (higher degree of anisotropy is possible if temperature is lower).

- It is highly unlikely that a high degree of electrical anisotropy extends pervasively in the crust.

- Even if lithospheric anisotropy exists, the most conductive direction in the anisotropic lithospheric structure should be sufficiently conductive (likely $<1000 \Omega \mathrm{m}$ ) to resolve a difference between it and an isotropic lithosphere using isotropic inversion.

\section{Additional file}

Additional file 1. The NoMelt data of Sarafian et al. (2015) and results of our 2D isotropic and anisotropic inversions of the data.

\section{Abbreviation}

MT: magnetotelluric

\section{Authors' contributions}

The authors conducted all of this study and wrote the manuscript. All authors read and approved the final manuscript.

\section{Author details}

${ }^{1}$ Kobe Ocean Bottom Exploration Center, Kobe University, Kobe, Japan. ${ }^{2}$ Earthquake Research Institute, The University of Tokyo, Tokyo, Japan.

${ }^{3}$ Department of Geology and Geophysics, Woods Hole Oceanographic Institution, Woods Hole, MA, USA.

\section{Acknowledgements}

Much of this work was carried out, while TM was appointed at the Ocean Hemisphere Research Center, Earthquake Research Institute, the University of Tokyo, and RLE visited the institute hosted by Kiyoshi Baba with support from the International Research Promotion Office of the institute. We are grateful to members at the institute for offering the opportunity to conduct this work. RLE was supported by NSF Grant OCE-0928663. We thank Kiyoshi Baba for his comments and Anne Pommier for providing comments on an early version of the manuscript and for giving us access to her manuscript detailing laboratory results prior to submission. We also thank James Gaherty, Greg Hirth, Daniel Lizarralde, and Pei-Ying Patty Lin for conversations that have shaped our thinking for this paper. We acknowledge Josef Pek for providing their forward modeling program. All the figures were produced using Generic Mapping Tool, GMT (Wessel et al. 2013). Comments and suggestions by two anonymous reviewers were helpful for improving the clarity of the manuscript.

\section{Competing interests}

The authors declare that they have no competing interests.

\section{Publisher's Note}

Springer Nature remains neutral with regard to jurisdictional claims in published maps and institutional affiliations.

Received: 28 April 2017 Accepted: 25 September 2017

Published online: 05 October 2017

\section{References}

Baba K, Chave AD, Evans RL, Hirth G, Mackie R (2006) Mantle dynamics beneath the East Pacific Rise at $17^{\circ} \mathrm{S}$ : insights from the mantle 
electromagnetic and tomography (MELT) experiment. J Geophys Res 111:B02101. doi:10.1029/2004JB003598

Beghein C, Yuan K, Schmerr N, Xing Z (2014) Changes in seismic anisotropy shed light on the nature of the Gutenberg discontinuity. Science 343:1237-1240. doi:10.1126/science.1246724

Behrens JP (2005) The detection of electrical anisotropy in 35 Ma Pacific lithosphere: results from a marine controlled-source electromagnetic survey and implications for hydration of the upper mantle. Ph.D. Thesis, University of California, San Diego

Blackman DK, Kendall JM (2002) Seismic anisotropy in the upper mantle. 2. Predictions for current plate boundary flow models. Geochem Geophys Geosyst 3(9):8602. doi:10.1029/2001GC000247

Blackman DK, Wenk H-R, Kendall JM (2002) Seismic anisotropy of the upper mantle. 1. Factors that affect mineral texture and effective elastic properties. Geochem Geophys Geosyst 3(9):8601. doi:10.1029/2001GC000248

Constable S (2006) SEO3: a new model of olivine electrical conductivity. Geophys J Int 166:435-437. doi:10.1111/j.1365-246X.2006.03041.x

Dai L, Kararo S (2014) High and highly anisotropic electrical conductivity of the asthenosphere due to hydrogen diffusion in olivine. Earth Planet Sci Lett 408:79-86. doi:10.1016/j.epsl.2014.10.003

Du Frane WL, Roberts JJ, Toffelmier DA, Tyburczy JA (2005) Anisotropy of electrical conductivity in dry olivine. Geophys Res Lett 32:L24315. doi:10. 1029/2005GL023879

Evans RL, Hirth G, Baba K, Forsyth D, Chave A, Mackie R (2005) Geophysical evidence from the MELT area for compositional controls on oceanic plates. Nature 437:249-252. doi:10.1038/nature04014

Everett ME, Constable S (1999) Electric dipole fields over an anisotropic seafloor: theory and application to the structure of $40 \mathrm{Ma}$ Pacific Ocean lithosphere. Geophys J Int 136(1):41-56. doi:10.1046/j.1365-246X.1999.00725.x

Gardés E, Gaillard F, Tarits P (2014) Toward a unified hydrous olivine electrical conductivity law. Geochem Geophys Geosyst 15:4984-5000. doi:10.1002 /2014GC005496

Heise W, Caldwell TG, Bibby HM, Brown C (2006) Anisotropy and phase splits in magnetotellurics. Phys Earth Planet Inter 158:107-121. doi:10.1016/j. pepi.2006.03.021

Key K, Constable S, Matsuno T, Evans RL, Myer D (2012) Electromagnetic evidence for plate hydration due to bending faults at the Middle America Trench. Earth Planet Sci Lett 351-352:45-53. doi:10.1016/j. epsl.2012.07.020
Lin P-YP, Gaherty JB, Jin G, Collins JA, Lizarralde D, Evans RL, Hirth G (2016) High-resolution seismic constraints on flow dynamics in the oceanic asthenosphere. Nature 535:538-541. doi:10.1038/nature18012

Montagner J-P, Guillot L (2002) Seismic anisotropy and global geodynamics. Rev Mineral Geochem 51:353-385

Naif S, Key K, Constable SC, Evans RL (2015) Water-rich bending faults at the Middle America Trench. Geochem Geophys Geosyst 16:2582-2597. doi:1 $0.1002 / 2015 G C 005927$

Nicolas A, Christensen NI (1987) Formation of anisotropy in uppermantle peridotites: a review. In: Froidevaux C, Fuchs K (eds) The composition, structure and dynamics of the lithosphere-asthenosphere system. American Geophysical Union, Washington, pp 111-123

Pek J, Santos FAM (2002) Magnetotelluric impedances and parametric sensitivities for 1-D anisotropic layered media. Comput Geosci 28(8):939-950. doi:10.1016/S0098-3004(02)00014-6

Poe BT, Romano C, Nestola F, Smyth JR (2010) Electrical conductivity anisotropy of dry and hydrous olivine at $8 \mathrm{GPa}$. Phys Earth Planet Inter 181:103-111. doi:10.1016/j.pepi.2010.05.003

Pommier A, Leinenweber K, Kohlstedt DL, Qi C, Garnero EJ, Mackwell SJ, Tyburczy JA (2015) Experimental constraints on the electrical anisotropy of the lithosphere-asthenosphere system. Nature 522:202-206. doi:10.1038/nature14502

Rodi W, Mackie RL (2001) Nonlinear conjugate gradients algorithm for 2-D magnetotelluric inversion. Geophysics 66:174-187

Sarafian E, Evans RL, Collins JA, Elsenbeck J, Gaetani GA, Gaherty JB, Hirth G, Lizarralde D (2015) The electrical structure of the central Pacific upper mantle constrained by the NoMelt experiment. Geochem Geophys Geosyst 16:1115-1132. doi:10.1002/2014GC005709

Tanimoto T, Anderson DL (1984) Mapping convection in the mantle. Geophys Res Lett 11(4):287-290. doi:10.1029/GL011i004p00287

Wessel P, Smith WHF, Scharroo R, Luis J, Wobbe F (2013) Generic mapping tools: improved version released, Eos, trans. AGU 94(45):409-420. doi:10.1 002/2013EO450001

Yoshino T, Matsuzaki T, Yamashita S, Katsura T (2006) Hydrous olivine unable to account for conductivity anomaly at the top of the asthenosphere. Nature 443:973-976. doi:10.1038/nature05223

\section{Submit your manuscript to a SpringerOpen ${ }^{\circ}$ journal and benefit from:}

- Convenient online submission

- Rigorous peer review

- Open access: articles freely available online

- High visibility within the field

Retaining the copyright to your article

Submit your next manuscript at $\boldsymbol{\nabla}$ springeropen.com 